\title{
Stabilization of ultra-lean hydrogen enriched inverted flames behind a bluff-body and the phenomenon of anomalous blow-off
}

\author{
Carmen Jiménez *a,b, Dan Michaels ${ }^{\mathrm{a}, \mathrm{c}}$, Ahmed F. Ghoniem ${ }^{\mathrm{a}}$ \\ ${ }^{a}$ Massachusetts Institute of Technology, 77 Massachusetts Avenue, Cambridge, MA \\ 02139, USA \\ ${ }^{b}$ CIEMAT, Avenida Complutense 40, 28040 Madrid, Spain \\ ${ }^{c}$ Technion-Israel Institute of Technology, Haifa 32000, Israel
}

\begin{abstract}
This paper presents a fundamental study of ultra-lean flames stabilized behind a thin, highly conducting metallic rectangular bluff body acting as a flame holder. Using high fidelity numerical simulations, we reproduce a phenomenon observed experimentally, showing that in this configuration steady hydrogen-methane flames can exist at equivalence ratios below the flammability limit associated with planar unstrained flames with the same hydrogenmethane proportion. These ultra-lean hydrogen-enriched mixtures exhibit a distinct stabilization mechanism compared to pure methane flames: they stabilize in the form of inverted closed $\mathrm{V}$ or $\mathrm{U}$ flames farther away from the flame holder as the inflow reactants velocity is reduced, leading eventually to blow-off for sufficiently small velocities. Conversely, as the reactants flow rate is increased, the flames anchor closer to the flame holder, and surprisingly no blow-off is observed at high velocities. This response is shown to be linked to
\end{abstract}

${ }^{*}$ Corresponding author, email address carmen.jimenez@ciemat.es 
the presence of hydrogen in the fuel mixture and its large diffusivity, which results in locally richer mixtures in the strained, curved flame base.

Keywords: premixed flames, stabilization, ultra-lean, conjugate heat exchange, hydrogen, preferential diffusion

\section{Introduction}

Flame anchoring (or static stabilization) using a solid obstacle as a flame holder is a recurrent subject of interest in combustion science, both as a fundamental research problem and because it is one of the practical means of effectively obtaining a stable premixed flame in a high velocity reactant flow over a wide range of inflow rates. The solid body immersed in the flow creates a low velocity recirculation zone where the flame can anchor even when the burning velocity is very small compared to the mean flow velocity. The recirculating flow produces a flux of hot products towards the reactants that contribute to their preheating, therefore favoring ignition conditions. Additionally, the solid body can contribute to flame stabilization by providing means by which some of the heat produced in the combustion zone is conducted through the solid back to preheat the reactants. Aerodynamic strain and curvature, together with possible effects of differential diffusion and heat transport determine the local flame propagation speed and its shape.

The relative relevance of these different stabilization mechanisms depends on the problem parameters, e.g., the flow velocity, fuel composition, bluffbody shape and material, etc. Stabilization, in some cases, can be achieved without thermal interaction with the flame holder (adiabatic stabilization $[1,2,3])$, while in others stability criteria depend on the thermal properties 
of the bluff-body [4]. As discussed in [4] and later studies [5, 6, 7], this depends on the mixture burning characteristics and flow rate as well as on the thermal properties of the solid. Thus, including conjugate heat exchange between the gas and the bluff-body is warranted unless it is clear a priori that this interaction is negligible.

Until recently, experiments have been the primary source of information on the study of bluff-body stabilized flames. Starting with the pioneering work of Lewis and von Elbe [8,9], who performed experiments of inverted flames stabilized behind a central body and linked the blow-off to the velocity gradient at the nozzle reaching a critical value, many researchers have tried to explain the mechanisms that affect flame stabilization and blow-off in these flames, adding other factors such as flame curvature $[2,3]$ and heat exchange with the flame holder $[1,2,3,10]$. A review on the dynamics of blow-off of turbulent flames stabilized behind a bluff-body can be found in [11].

Numerical studies where the combustion field, its heat exchange with the solid body and the internal temperature distribution inside the latter can be coupled have been scarce until recent years. Advances in computational power and parallel computing as well as specific time-coupling strategies $[12$, 13] have allowed direct numerical simulations of this kind of problems using detailed chemistry and transport properties of complex fuels and including the conjugate heat exchange with the solid $[4,5,6,7,14,15]$.

Most of the studies mentioned above have focused on mechanisms of methane flames stabilization behind solid bodies. A recent interesting experimental study [16] (also studied numerically in [17]), has, however, shown a different stabilization mechanism of hydrogen-enriched flames. That work 
examined methane-air and methane-hydrogen-air flames anchored behind a long, highly conducting metallic cylindrical rod with a small diameter (1 to $3 \mathrm{~mm}$ ), placed along the axis of a circular channel. It showed that pure methane flames behave according to the well-know mechanism, anchoring farther away from the solid rod as the flow rate is raised and eventually blowing off at a high enough flow rate. On the other hand, when the burning mixture contains a sufficient amount of hydrogen, the trend is reversed: increasing the flow rate results in the flame anchoring closer to the metallic rod and reducing the flow rate leads to flames stabilized farther from the flame holder and can eventually lead to blow-off. This blow-off by decreasing the inflow velocity, termed "anomalous blow-off" in [16], was found when hydrogen content was equal or larger than $20 \%$. For these mixtures "normal blow-off", that is, flame extinction as the flow rate is raised, at high flow rates was also reported. Moreover, the study showed that in this configuration hydrogen-containing flames could be stabilized for mixtures well below the flammability limit, permitting ultra-lean combustion.

The authors relate this anomalous behavior to the effect of differential diffusion on the burning speed of a stretched methane-hydrogen flame. According to theory (see e.g. $[18,19]$ ), the influence of flame stretch on the flame burning speed is twofold: one is the contribution of pure stretch, which always decreases the flame speed (for positive stretch) and the second is the contribution of the combined differential diffusion (unequal heat and mass diffusivity) and stretch, whose effect on the flame speed depends on the mixture Lewis number. For mixtures with Lewis number smaller than one, differential diffusion results in more intense burning when positive stretch is 
increased. Mixtures with Lewis number larger than one show the opposite behavior, with the flame speed decreasing with increasing stretch. While it is difficult to define a global Lewis number for a mixture of methane-hydrogen, it is clear that for a sufficient fraction of hydrogen it should be smaller than one and the flame speed should increase with stretch.

Anomalous anchoring and blow-off was explained as follows: for sufficient hydrogen content the Lewis number is smaller than one and the burning intensity increases with flame stretch; therefore as the inflow velocity and consequently the flame stretch are raised the flame burns faster and moves closer to the flame holder. Moreover, to burn $L e<1$ fuel mixtures below their flammability limit, a minimum stretch rate is needed to increase the burning velocity and make the mixture flammable. What remains unclear is that for the same hydrogen content (and Le number) a mixture can also be blown off for very large stretch rates, as the experiments suggest (normal blow-off). The experimental study in [16] reported only measurements of the flame position change and the flame holder temperature, no measurements of the flame stretch rate or burning speed were available, hence validation of the hypothesis described above was not feasible. Moreover, the analysis in [17] was mainly devoted to the study of flame stability and not to explaining the anomalous blow-off mechanism.

The aim of the present work is to contribute to the study of this problem using detailed numerical simulations, from which more detailed information can be extracted. First, we attempt to reproduce the blow-off phenomenon. For simplicity we selected to perform the simulations in a planar (2D) setup, with a fixed-size, shorter flame holder and for mixtures with a single 
value of methane-hydrogen ratio. After experimenting with different bluffbody sizes and reactant mixtures we selected a flame holder with height $d$ equal to $5 \mathrm{~mm}$ and length equal to $3 d$, and a mixture with $\mathrm{H}_{2}-\mathrm{CH}_{4}$ ratio 40\%-60\%. For this hydrogen fraction, ultra-lean flames and anomalous blowoff were reported in experiments (albeit in the experiment the solid body is cylindrical and these effects were observed for diameters $\geq 2 \mathrm{~mm}$ ). Next, by varying the equivalence ratio of the mixture and/or the reactants flow rate we determined the limits for flame stabilization, finding that blow-off occurs as the velocity was reduced, for ultra-lean mixtures with $\phi=0.35$. This study utilizes direct numerical simulation (DNS) of chemically reacting flows in the presence of a heat conducting solid developed by Kedia et al. [20]. As discussed in our previous publications, our model includes conjugate heat transfer between the fluid and the solid, which is treated using an immersed boundary approach, a block-structured adaptive mesh refinement strategy to adapt to local flame resolution needs and detailed chemical kinetics and species transport.

In section 2 we describe briefly the conservation equations and the numerical method; section 3 introduces the particular set-up used to model the present problem; in section 4 we describe the steady flame solutions, and characterize the heat exchange with the flame holder, the flame stretch rate and the effect of the large hydrogen diffusivity in flame stabilization. Finally, section 7 summarizes the main conclusions of our study. 


\section{Numerical model}

Under the low-Mach number approximation, the conservation equations for mass, momentum, energy and species are:

$$
\begin{aligned}
& \frac{\partial \rho}{\partial t}=-\nabla \cdot(\rho \mathbf{v}) \\
& \frac{\partial \mathbf{v}}{\partial t}=-\frac{1}{\rho} \nabla p+C_{U}+D_{U} \\
& \frac{\partial T}{\partial t}=C_{T}+D_{T}+S_{T} \\
& \frac{\partial Y_{k}}{\partial t}=C_{Y_{k}}+D_{Y_{k}}+S_{Y_{k}}, k=1, \ldots, N_{s}
\end{aligned}
$$

where $\mathbf{v}$ is the velocity vector, $\rho$ the density, $T$ the temperature, $Y_{k}$ the $k$ species mass fraction, $p$ the hydrodynamic pressure and $N_{s}$ the number of involved chemical species. This system of equations is supplemented with the equation of state for an ideal gas:

$$
p_{0}=\frac{\rho \mathcal{R} T}{\bar{W}},
$$

where $p_{0}$ is the thermodynamic pressure, considered spatially uniform in the low-Mach number limit, and also constant in time in the present, opendomain configuration; $\mathcal{R}$ stands for the universal gas constant, and $\bar{W}$ corresponds to the mixture molecular weight: $\frac{1}{\bar{W}}=\sum_{k=1}^{k=N_{s}} \frac{Y_{k}}{W_{k}}$, with $W_{k}$ the molecular weight of species $k$.

The convection, diffusion and reaction terms in Eqs. 1 are:

$$
C_{U}=-(\mathbf{v} \cdot \nabla) \mathbf{v} ; \quad D_{U}=\frac{1}{\rho} \nabla \cdot \tau ;
$$




$$
\begin{array}{r}
C_{T}=-(\mathbf{v} \cdot \nabla) T ; \quad D_{T}=\frac{1}{\rho c_{p}} \nabla \cdot(\lambda \nabla T)-\left(\sum_{k=1}^{N_{s}} c_{p, k} Y_{k} \mathbf{V}_{k}\right) \cdot \nabla T ; \\
S_{T}=-\frac{1}{\rho c_{p}} \sum_{k=1}^{N_{s}} h_{k} \dot{\omega}_{k} ; \\
C_{Y_{k}}=-(\mathbf{v} \cdot \nabla) Y_{k} ; \quad D_{Y_{k}}=-\frac{1}{\rho} \nabla \cdot\left(\rho Y_{k} \mathbf{V}_{k}\right) ; \quad S_{Y_{k}}=\frac{1}{\rho} \dot{\omega}_{k} .
\end{array}
$$

In the expressions above $\tau$ is the stress tensor of components:

$$
\tau_{i j}=\mu\left(\frac{\partial u_{i}}{\partial x_{j}}+\frac{\partial u_{j}}{\partial x_{i}}-\frac{2}{3} \delta_{i j} \nabla \cdot \mathbf{v}\right),
$$

with $\mu$ the dynamic viscosity of the mixture and $u_{i}$ the components of the velocity vector; $\mathbf{V}_{k}$ is the diffusion velocity of species $k$, modeled as:

$$
\mathbf{V}_{k}=-\frac{D_{k, m}}{X_{k}}\left(\nabla X_{k}\right)-\frac{D_{k}^{T}}{\rho Y_{k}} \frac{\nabla T}{T}
$$

where $X_{k}$ stands for the mole fraction of species $k, D_{k, m}$ is the mixtureaveraged diffusivity of species $k$ and $D_{k}^{T}$ is its thermal diffusion coefficient, which is introduced to account for the Soret effect (or thermal diffusion) of light species. To ensure global mass conservation the last species mass fraction, here $Y_{\mathrm{N}_{2}}$, is calculated from $\sum_{k=1}^{k=N_{s}} Y_{k}=1$. This is an alternative to introducing a correction velocity in the expression of the diffusion velocities above, and is valid only if, as $\mathrm{N}_{2}$ in the present case, there is a dominant diluent with a large concentration[19]. Moreover, $c_{p, k}$ and $c_{p}$ stand for the specific heat at constant pressure for species $k$ and for the mixture, respectively, $\lambda$ is the mixture thermal conductivity and $h_{k}$ and $\dot{\omega}_{k}$ represent the molar enthalpy and molar production rate (per unit time and volume) of 
species $k$. The NASA polynomials [21] are used to compute the thermodynamic properties of the mixture; the mixture-averaged transport properties are evaluated using a dipole-reduced formalism [22].

The conjugate heat exchange between the solid and the fluid is incorporated by simultaneously integrating the conservation equations for the reacting flow, Eqs. 1, and the transient heat conduction equation in the solid body:

$$
\frac{\partial T}{\partial t}=\frac{1}{\rho_{s} c_{p s}} \nabla \cdot\left(\lambda_{s} \nabla T\right)
$$

where $\rho_{s}, c_{p s}$ and $\lambda_{s}$ are, respectively, the density, the heat capacity and the thermal conductivity of the solid material. A no-penetration condition, the continuity of the temperature field and the conservation of heat flux are imposed at each time step in the solid-fluid boundary using the buffer-zone method presented in [20].

The numerical integration of the equations presented above is performed using the automatic mesh refinement method proposed in [23]. A projection approach is adopted first for the momentum equations in a fixed uniform mesh. In a second phase a symmetric Strang splitting approach is recursively implemented for the chosen hierarchy of automatic mesh refinement levels. Finally the projection step is repeated using the updated scalar fields.

\section{Numerical simulation set-up}

Figure 1 presents a sketch of the geometry of the present problem. The flame holder, shaded in black in the figure, is a rectangular body of width $d=5 \mathrm{~mm}$ and length $l=3 d$. The computational domain is a channel of height $h=5 d$ and length $L=12.5 d$. The flame holder is considered to be 


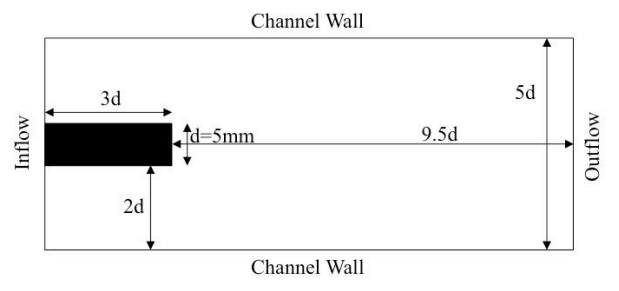

Figure 1: A sketch of the computational domain.

a highly conducting metal with $\lambda_{s}=60 \mathrm{~W} /(\mathrm{m} \mathrm{K})$ and $\rho_{s} c_{p s}=56532 \mathrm{~J} /\left(\mathrm{m}^{3}\right.$ $\mathrm{K})$. It should be mentioned that this value of the heat capacity times the density of the solid material only affects the time derivative of temperature (see Eq. 6). It does not have any influence on the steady state solution of the system of equations. For this reason it was often decreased during transitional stages in our simulations to accelerate the convergence towards the steady solution.

The fuel composition is $40 \% \mathrm{H}_{2}$ and $60 \% \mathrm{CH}_{4}$ by volume. For this fraction of hydrogen, ultra-lean combustion and anomalous blow-off were reported in the experiments in [16]. The fuel is premixed with air to create mixtures at equivalence ratios $\phi$ between 0.3 and 0.4. The equivalence ratio is defined according to the stoichiometric relation:

$$
\phi=\frac{1}{2} \frac{X_{\mathrm{H}_{2}}}{X_{\mathrm{O}_{2}}}+2 \frac{X_{\mathrm{CH}_{4}}}{X_{\mathrm{O}_{2}}},
$$

where $X_{k}$ stands for the mole fraction of species $k$. The skeletal chemical kinetics scheme proposed in [24], consisting of 46 reversible reactions among 16 species is used to compute the species production rates. This mechanism has been validated for lean $\mathrm{CH}_{4} / \mathrm{H}_{2}$ flames in [14]. The lean flammability limit obtained for a $40 \% \mathrm{H}_{2}-60 \% \mathrm{CH}_{4}$ planar unstrained flame using this 
chemical kinetics is $\phi=0.425$, so that all the mixtures used in the present computations are below this flammability limit. If strain is applied to the planar flame the flammability limit is extended. The strained flames with $\phi<$ 0.425 are, however easily extinguished if strain is increased, so that they exist within a very small range of strain rates. For $\phi=0.35$ and $40 \% \mathrm{H}_{2}-60 \% \mathrm{CH}_{4}$, the minimum strain rate for a planar flame to exist is $\kappa=11 \mathrm{~s}^{-1}$, while the extinction strain rate is as low as $\kappa=31 \mathrm{~s}^{-1}$. Both quantities were estimated using the axisymmetric opposed-jet premixed flame model in CHEMKIN [25] and the above chemical kinetics mechanism.

Boundary conditions at the inflow of the computational domain correspond to a developed Poiseuille flow above and below the solid body with mean velocity $U$, reactant mass fractions given by $\phi$ and pressure and temperature conditions $p_{0}=1 \mathrm{~atm}, T_{0}=300 \mathrm{~K}$, so that the total mass flux at the inlet is $\dot{m}=\rho_{0} U 4 d$ with $\rho_{0}$ given by Eq. $2 . U$ is varied between $5 \mathrm{~cm} / \mathrm{s}$ and 8.5 $\mathrm{m} / \mathrm{s}$, resulting in a Reynolds number variation from $R e_{d}=15$ to $R e_{d}=2590$, based on the flame holder width $d$. At the outlet, "convective-flow" boundary conditions are used for the velocity and scalars and a Neumann (zerogradient) condition is applied to the pressure [23]. No-slip, impermeable, adiabatic conditions are imposed at the channel walls, while the left wall of the solid body was chosen to be isothermal, at ambient temperature equal to $T_{0}=300 \mathrm{~K}$. This condition, together with the developed channel flow at the inlet of the domain, are chosen to model a quasi-isothermal long planar, cooled bluff-body, somewhat different fom the long body of the experiment.

Note that this boundary condition will involve global heat losses in the computational domain, and as such, will represent the worst-case condition 
for flame stabilization. Nevertheless, we will show that these heat losses are very small compared to the rate of heat production in the steady inverted flames, and will not have an impact on flame stabilization. Radiation heat losses are neglected on account of the low temperatures reached by the gas and the flame holder.

To verify that the boundary condition at the left wall of the flame holder does not significantly affect flame stabilization, we conducted additional simulations using a rectangular solid body of length $l=6 d$. Differences in the computed flames are negligible, as can be verified in Fig. 2, which compares temperature profiles along the central $x$-axis obtained for three different inflow velocities using a flame holder of length $3 d$ (solid lines) and $6 d$ (dots). Changes in the measured flame stand-off distance, the recirculation zone length or the temperature at the flame holder tip amount to a maximum of $3 \%$. It should be mentioned that the fact that changes are negligible is related to the high conductivity of the solid material.

The size of the computational grid was chosen to be $\Delta x=\Delta y=196 \mu \mathrm{m}$, and one additional refinement level in the adaptive mesh refinement was allowed. This means that the flame resolution was increased to $98 \mu \mathrm{m}$ where needed. The flame thickness for the planar unstrained flame at $\phi=0.425$ mentioned above can be estimated from the mixture properties and the laminar flame speed $S_{L}$ to be $\delta_{T}=\lambda /\left(\rho c_{p} S_{L}\right) \approx 1.5 \mathrm{~mm}$. Therefore the chosen mesh resolution results in nearly 15 points inside the flame thickness of a planar flame near the flammability limit. Given that the ultra-lean flames under the flammability limit of the present study are slowlier and consequently thicker, the same level of resolution should also suffice. 


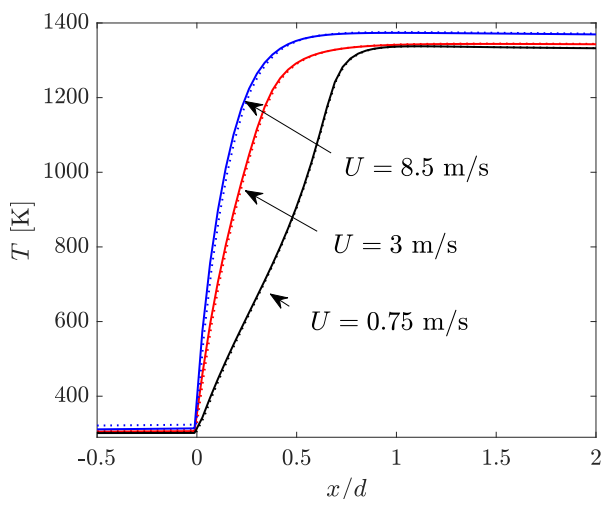

Figure 2: Comparison of the temperature profiles along the central axis for three steady flames at different inflow velocities for a model flame holder of length $3 d$ (solid lines) and $6 d$ (dotted lines).

\section{Results and discussion}

Following the establishment of a steady flame, by varying the mixture equivalence ratio $\phi$ and the reactants inflow rate $U$, we covered the full range of stable flames between $\phi=0.35$ and $\phi=0.4$. Figure 3 shows the distance between the flame base and the solid body, that is, the stand-off distance, for flames with $\phi=0.35$ and $\phi=0.4$ at different $U$. All these cases correspond to steady flames. We note that the exact value of this anchoring distance depends on the definition of the flame location. In this work we define the flame surface as the surface where the heat release rate is $10 \%$ of the maximum heat release rate.

It is clear from Fig. 3 that for the two selected mixtures, as the reactant mass flow rate is raised, the flame anchors at a point closer to the flame holder. For the highest inflow rates the stand-off distance is as small as $0.18 d(0.9 \mathrm{~mm})$. Conversely, as $U$ is decreased, a steady flame stabilizes 


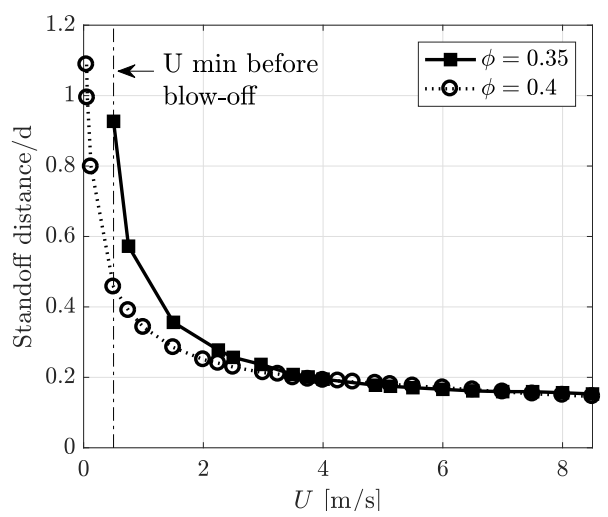

Figure 3: The change of the distance from the flame base to the flame holder (stand-off distance) for flames with $40 \%$ hydrogen and $60 \%$ methane at equivalence ratios $\phi=0.35$ and 0.40 as a function of the reactants inflow velocity $U$.

farther away from the flame holder, with the farthest flames corresponding to stand-off distances of about $0.9 d=4.5 \mathrm{~mm}$ for the leaner case $(\phi=0.35)$ and $1.1 d=5.5 \mathrm{~mm}$ for the richer case $(\phi=0.4)$.

In the case at equivalence ratio $\phi=0.35$ the minimum inflow velocity for which a steady flame exists is $U=0.5 \mathrm{~m} / \mathrm{s}$; reducing this velocity leads to flame blow-off. Thus we can reproduce the phenomenon observed experimentally [16] for the case with $\phi=0.35$ : a minimum inflow velocity is needed to establish a steady flame and the anchoring distance decreases as the inflow velocity is increased. This is what was called "anomalous blow-off" [16] and is opposite to what is observed in methane flames, in which the stand-off distance grows as the reactants flow increases until blow off occurs at sufficiently high flow speed. For the mixture with $\phi=0.4$, no blow-off was found even when the inflow reactants velocity was reduced to a value as low as $U=0.05 \mathrm{~m} / \mathrm{s}$. This mixture is very close to the flammability limit and no 

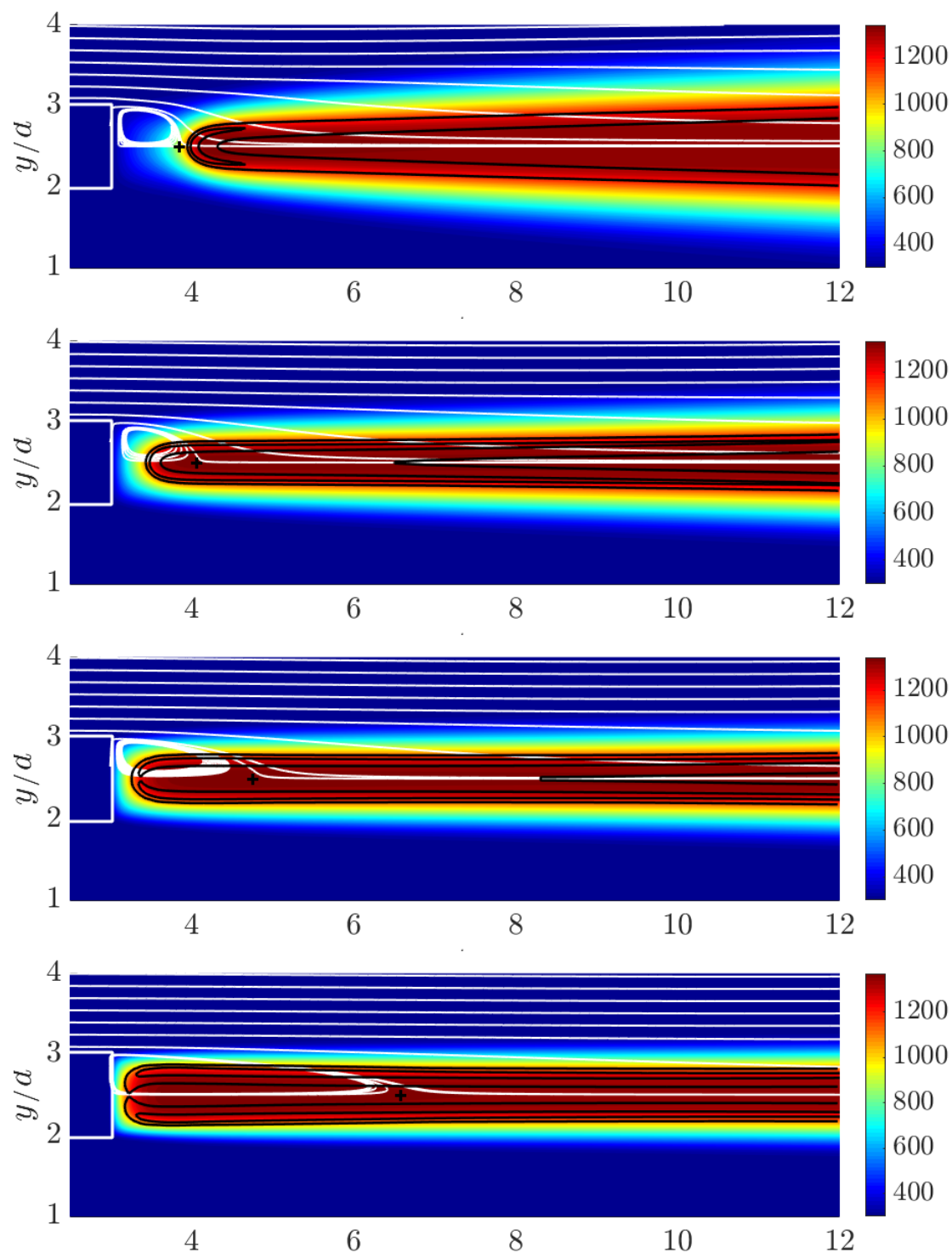

Figure 4: Steady flames corresponding to a mixture with $40 \% \mathrm{H}_{2}-60 \% \mathrm{CH}_{4}$ and $\phi=0.35$ and to inflow reactant velocity $U=0.5,1.5,3$ and $8.5 \mathrm{~m} / \mathrm{s}$. The color contour plots show the temperature distribution. The black solid line marks the flame surface location (corresponding to $10 \%$ of the maximum heat release) and a second heat release contour at $50 \%$ of the maximum. Selected streamlines are plotted in white to visualize the recirculation zone, while the + sign corresponds to the stagnation point at the end of the recirculation zone. 


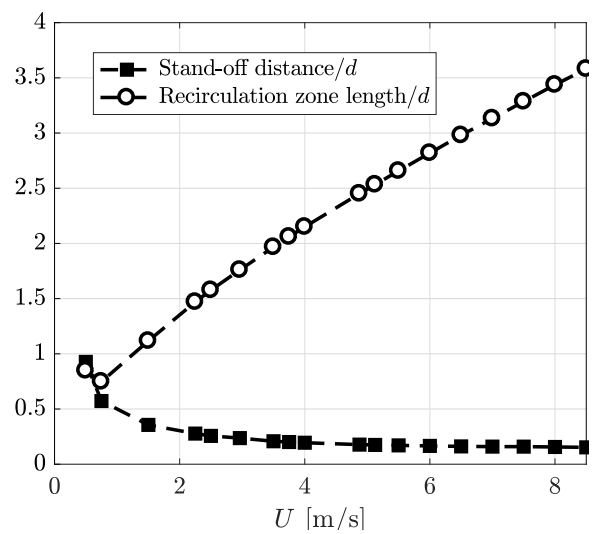

Figure 5: The stand-off distance compared to the recirculation zone length for flames with equivalence ratio $\phi=0.35$ as a function of the reactants inflow velocity $U$.

anomalous blow off was found. We restrict the rest of our study to mixtures with equivalence ratio equal or lower than $\phi=0.35$.

To investigate the flame stabilization mechanism, we will first study the shape change as the reactant flow velocity is increased. In the experiments in [16], only video images and temperature measurements were reported and no detailed flow measurements were available. The flames were interpreted as inverted $\mathrm{U}$ or $\mathrm{V}$ flames anchored just downstream the recirculation zone. This implied also that the flame stretch produced by the flow should be negative. In the present study we have access to all the variables involved in the flame and flow dynamics and hence can determine the relative location of the flame with respect to the flow streamlines and the sense of stretch. To visualize the different flame shapes, contour plots of the temperature field and the reaction zone location are presented in Fig. 4 for four selected cases, corresponding to steady flames with $\phi=0.35$ and inflow reactant velocities 
$U=0.5 \mathrm{~m} / \mathrm{s}$ (the slowest flow for which a steady flame was obtained for this equivalence ratio), $1.5 \mathrm{~m} / \mathrm{s}, 3 \mathrm{~m} / \mathrm{s}$ and $8.5 \mathrm{~m} / \mathrm{s}$ (the highest value of $U$ in the present study). The flow is in all cases from left to right. All the flames are symmetric with respect to the flame holder axis. The colored contours show the temperature distribution, while black solid lines mark isosurfaces of heat release rate equal to $10 \%$ (corresponding to the flame surface) and $50 \%$ of the maximum heat release rate. Selected streamlines are plotted in white to visualize the recirculation zone, and the + sign corresponds to the stagnation point at the end of the recirculation zone.

The plots in Fig. 4 show that for the slowest flow the recirculation bubble is very small and the flame anchors just downstream the + sign marking its end. As will be seen later, since the converging flow creates a compression effect at the flame base, this corresponds to negative strain-induced flame stretching at that location. However, as the flow rate is raised, the recirculation zone grows while at the same time the flame moves closer to the flame holder. This can also be seen in Fig. 5, where the stand-off distance and the length of the recirculation zone for flames with varying inflow velocity $U$ are compared. For $U>0.5 \mathrm{~m} / \mathrm{s}$ all the flames anchor inside the recirculation zone. In these cases, the flame strain-induced stretch at the base is positive and increases as the flow rate increases. This is in contrast to pure methane flames in [4], in a similar configuration, which were shown to always stabilize outside the recirculation zone and for which the stretch rate linked to flow strain at the flame base was always negative. Moreover, at the highest flow velocity in Fig.4 the flame is not anchored at the central axis and instead stabilizes at two locations away from the central axis and still inside the re- 
circulation zone. This is very likely the result of a strong reverse flow along the central axis. The flame shape and location therefore change as the flow rate is increased: from a flame stabilizing just downstream the recirculation zone as was depicted in [16] to a flame stabilizing inside the recirculation zone with the flame base located along the central axis, to a flame consisting in two symmetric flame branches located inside the recirculation zone at some distance off the central axis.

We increased the reactants inflow velocity to values up to $U=8.5 \mathrm{~m} / \mathrm{s}$ and did not find any blow off at high velocity: as $U$ is raised the flame simply moves closer to the flame holder, and is firmly anchored to it, with no sign of it extinguising or blowing away. In the experiments in [16], blow-off was observed when increasing the inflow velocity, at values of about $U=3 \mathrm{~m} / \mathrm{s}$; this was what the authors called "normal blow-off". Several explanations could be proposed at this point to account for the different observations in the experiment and the simulations: 1) heat losses could be important in the experiments, leading to extinction as the flame moves to a location closer to the metallic body; 2) very high stretch rates at the flame base as $U$ is increased could result in flame extinction that our simulations can not reproduce (either because of inadequate chemical kinetics or because of the planar configuration); 3) turbulent fluctuations might be present in the experiments at the highest inflow rates, which could lead to extinction. (In the current numerical simulations, since changes in velocity are smooth, no perturbation is introduced and the numerical flow remains laminar for the full range of velocities shown in Fig. 3.) We will investigate these possible explanations in what follows. 


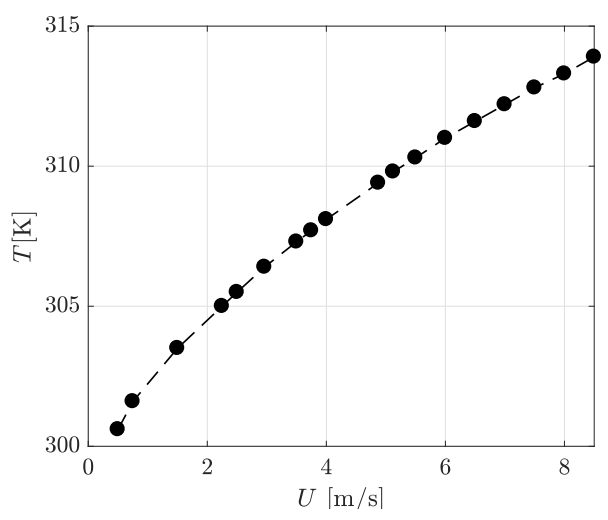

Figure 6: The maximum temperature in the solid flame holder for increasing inflow velocity $U$.

\subsection{Heat losses to the flame holder}

As seen in Figs. 3 and 4, when the flux of reactants is increased the flame moves closer to the flame holder. This should then make the heat exchange with the flame holder more important as the inflow reactant velocity $U$ grows.

Figure 6 presents the maximum temperature in the solid body as $U$ is increased. The solid's temperature grows with $U$ but temperature rises of only about $15 \mathrm{~K}$ above ambient are measured for the highest values of $U$. This value is similar to that reported in the experiments in [16], where the maximum measured temperature rise in the metallic rod was about $12 \mathrm{~K}$ (or even smaller, depending on the fuel composition). The low temperature rise is the result of competition of solid heating by the flame and cooling by fresh gases via the lateral and left walls. Because of the high thermal conductivity, cooling is strong and the solid tip remains at a temperature close to ambient, our modeled flame holder is therefore quasi-isothermal. Note that at this high conductivity, when the solid body length (and therefore the thermal exchange 
(a)

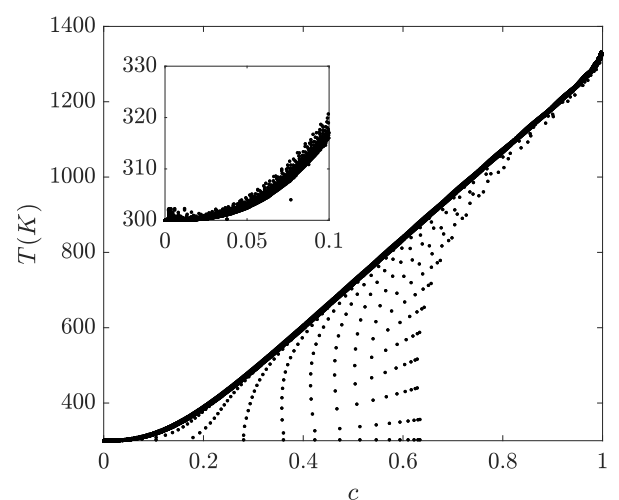

(b)

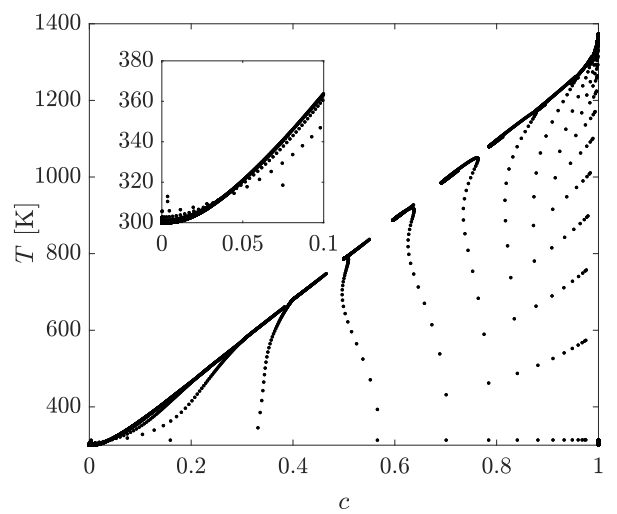

Figure 7: Scatter plot (phase plane representation) of the gas temperature $T$ versus a progress variable $c=1-\frac{Y_{\mathrm{H}_{2}}+Y_{\mathrm{CH}_{4}}}{Y_{\mathrm{H}_{2}}^{0}+Y_{\mathrm{CH}_{4}}^{0}}$ for two steady flames with $\phi=0.35$ and (a) $U=1.5$ $\mathrm{m} / \mathrm{s}$ and (b) $U=8.5 \mathrm{~m} / \mathrm{s}$. 
(a)

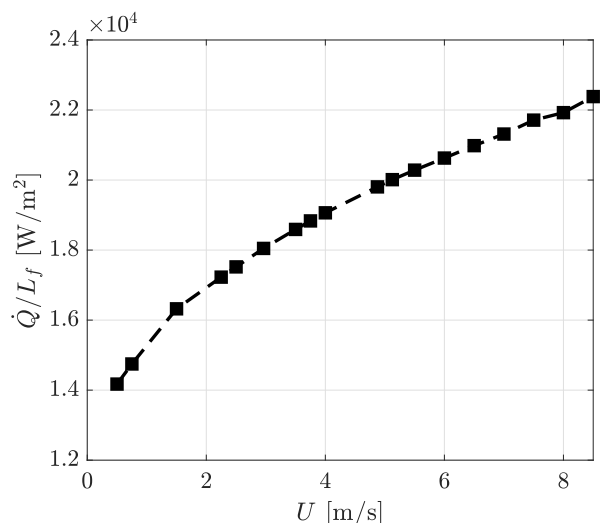

(b)

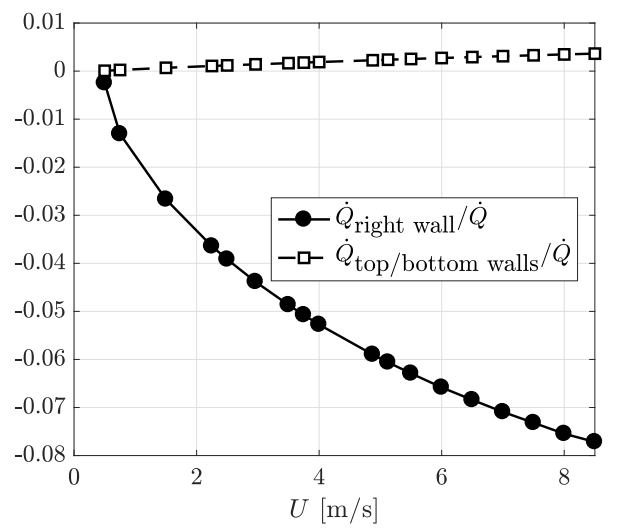

Figure 8: (a) The total heat release rate (per unit length in $z$ direction and scaled with the flame length $L_{f}$ ), and (b) the fraction of heat lost by conduction to the solid flame holder $\left(\dot{Q}_{\text {right wall }} / \dot{Q}\right)$ and that recovered via the top/bottom walls $\left(\dot{Q}_{\text {top/bottom walls }} / \dot{Q}\right)$ for increasing reactant flow velocity $U$. 
surface) was doubled (in the validation simulations as explained in section 3 ), the maximum flame holder temperature increased by only $3 \%$.

The impact of heat losses can be seen by plotting the gas composition and temperature over the whole computational domain, as in the scatter plots (phase plane) of Fig. 7. In this figure, the local temperature at each point in two steady flame simulations is plotted against the local value of a progress variable, $c$, defined as the sum of the reactants mass fractions, scaled by its value in the fresh reactants stream: $c=1-\frac{Y_{\mathrm{H}_{2}}+Y_{\mathrm{CH}_{4}}}{Y_{\mathrm{H}_{2}}^{0}+Y_{\mathrm{CH}_{4}}^{0}}$. With this definition $c=0$ in the fresh reactants and $c=1$ in the combustion products. The two scatter plots in Fig. 7 correspond to flames with $\phi=0.35$ and $U=1.5$ $\mathrm{m} / \mathrm{s}$, a moderate inflow velocity, for which the stand-off distance is $1.8 \mathrm{~mm}$ and $U=8.5 \mathrm{~m} / \mathrm{s}$, the highest inflow velocity in the current study, for which the flame is very close to the flame holder, at a distance of $0.8 \mathrm{~mm}$. It is evident from the scatter plots that the reactants preheating is very weak. For fresh reactants $(c \approx 0)$, the temperature is close to $300 \mathrm{~K}$, and it only increases significantly as $c$ grows, that is, as reactants are either consumed or mixed with products. Even in the case where the flame is closest to the flame holder (Fig. 7b) the temperature in the fresh reactants remains close to $300 \mathrm{~K}$; the insert in this figure shows temperature increases below $10 \mathrm{~K}$ in the $c=0$ region, showing that the reactants are only preheated by this small amount. The large scatter in the plots is a result of the heat transfer between products and reactants, as evidenced by points with $T=300 \mathrm{~K}$ but $c>0$.

Furthermore, Fig. 8a shows the total heat release rate per unit length of the flame, computed as the integral of the source term in Eq.1c scaled by the 
length of the flame $L_{f}$ in the corresponding simulation (because of changes in the stand-off distance the flame length included in the computational domain can vary appreciably). The heat release rate $\dot{Q} / L_{f}$ nearly doubles as the inflow reactants velocity is increased. Since the increase in the velocity leads to flames anchoring closer to the solid, we expect the total heat flux from the gas to the flame holder to grow as well with $U$. In Fig. 8b, it is evident that the fraction of heat that is transferred to the solid grows with $U$, but it is only a small percentage of the total heat release rate, with a maximum of only $8 \%$ for the largest reactant flux. Part of this heat is recovered via the lateral walls and is used to raise the reactants temperature but it is again a small percentage, amounting to only about $4 \%$ of the latter, or $0.5 \%$ of the total heat produced in the flame, as shown also in Fig. 8b. When the flame holder length was doubled, modeling cooling of the bluff-body further away from its end, the heat exchanged with the fresh gases was also nearly doubled. But still less than $8 \%$ of the heat is recovered and preheating remains negligible (below $10 \mathrm{~K}$ ). Even for a long flame holder, for which most of the heat could be recovered, the preheating of the reactants will remain low because of the high thermal conductivity of the body, which remains at temperatures close to ambient.

Even in this very unfavorable case where the high thermal conductivity and isothermal boundary condition result in a cold flame holder tip, which prevents the flame from locating too close to it, and where preheating of the gas via heat recirculation through the solid body is negligible, the flames are apparently impossible to blow off by increasing the flow velocity. Thermal losses to the conducting flame holder do not seem to be significant in the 
simulated flames. In the experiments [16], the maximum measured temperature rise in the metallic rod was of the same order of magnitude as in our simulations. In view of the very small heat losses to the flame holder, the blow-off for these steady flames is very unlikely to be related to the thermal interaction with the metallic rod as the flame approaches its surface.

\subsection{Flame stretch rate and flame structure}

The study of the structure and propagation of premixed flames is frequently based on the comparison to the classical model of the steady planar flame propagating against a fresh reactant mixture at a speed $S_{L}^{0}$, the laminar burning velocity[18]. Flames propagating in a non-uniform flow experience changes in the flame surface area, linked to flow strain and flame curvature. These changes can be measured by the stretch rate $\kappa$, defined as the variation in the flame surface area $A$ of an infinitesimal flame element: $\kappa=\frac{1}{A} \frac{d A}{d t}$. Asymptotic theory and experimental measurements suggest that for small stretch rates the difference between the curved flame speed and the corresponding laminar flame speed can be related linearly to the stretch rate $\kappa$ via a parameter $L_{a}$ known as the Markstein length:

$$
S_{c}=S_{L}^{0}-L_{a} \kappa
$$

Explicit expressions for the Markstein length have been derived from asymptotic analysis, showing that it is of the order of magnitude of the flame thickness, and depends also on the thermal expansion coefficient, the activation energy, and the deficient reactant Lewis number $L e_{D}[26,27]$. The sign of the Markstein length depends on the deficient reactant Lewis number such that $L_{a} \propto\left(L e_{D}-1\right)$ so that for $L e_{D}<1$ the Markstein length 
(a)

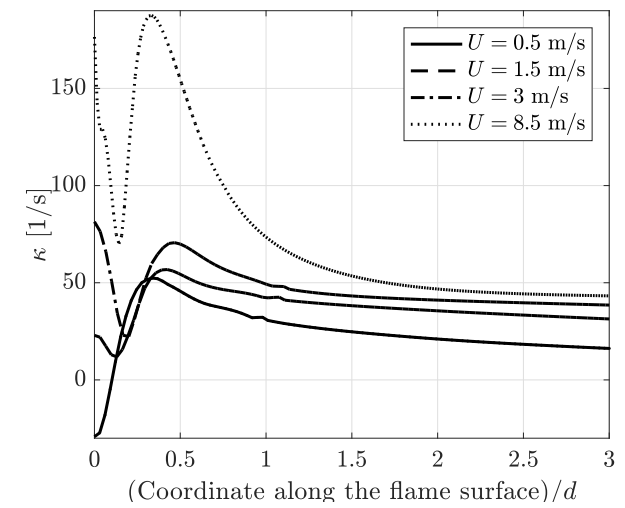

(b)

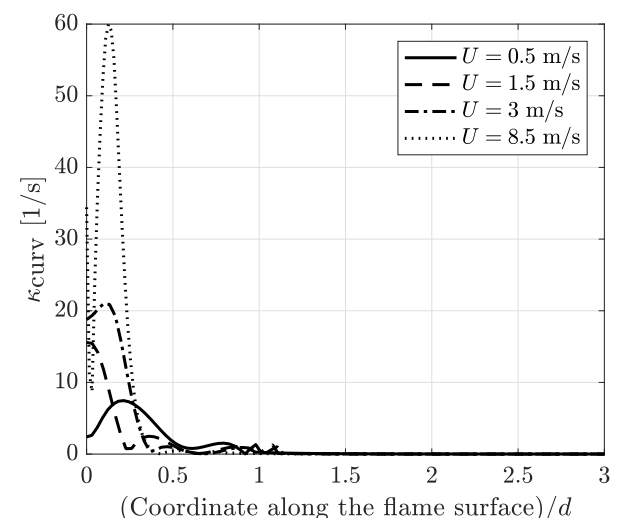

Figure 9: (a) The total flame stretch rate, $\kappa$ and (b) the curvature-related stretch $\kappa_{\text {curv }}$ along the flame surface for several flames with $\phi=0.35$ and different inflow reactant velocity $U$. 
is negative and therefore flame stretch should contribute to increasing the flame burning speed, according to Eq.8. In the current study, because of the presence of hydrogen in the fuel lean mixture, we can assume that the mixture Lewis number is smaller than one, and expect therefore a negative Markstein length so that the flame burning speed increases with stretch.

We measure the flame stretch rate along the steady flame surface as $[28$, 19]:

$$
\kappa=-n_{i} n_{j} \frac{\partial u_{i}}{\partial x_{j}}+\frac{\partial u_{i}}{\partial x_{i}}+S_{d} \frac{\partial n_{i}}{\partial x_{i}}
$$

where $n_{i}$ are the components of the unit vector normal to the flame (defined as positive when pointing towards the reactants), and $S_{d}$ is the flame displacement speed, computed as [19]:

$$
S_{d}=\frac{S_{T}+D_{T}}{|\nabla T|}
$$

Here $S_{T}$ and $D_{T}$ are the reaction and diffusion terms in the energy equation introduced in Eq. 1c. The first two terms in Eq. 9 correspond to stretch created by flow strain while the last term is related to the flame curvature.

In Fig. 9 we plot the total flame stretch, $\kappa$, and the curvature contribution, $\kappa_{\text {curv }}$, along the flame surface for four steady flames with $\phi=0.35$ and increasing flow rate $U$. The flame surface was defined as the isosurface of $T=1000 \mathrm{~K}$, which is close to the contour where the heat release rate amounts to $10 \%$ of its maximum value. For the flame corresponding to the slowest flow $(U=0.5 \mathrm{~m} / \mathrm{s})$, the flame stretch rate is negative near the flame base. This should be expected from the converging velocity field, which induces compression on the flame surface located downstream the recirculation zone. The stretch becomes positive downstream along the flame surface. The 

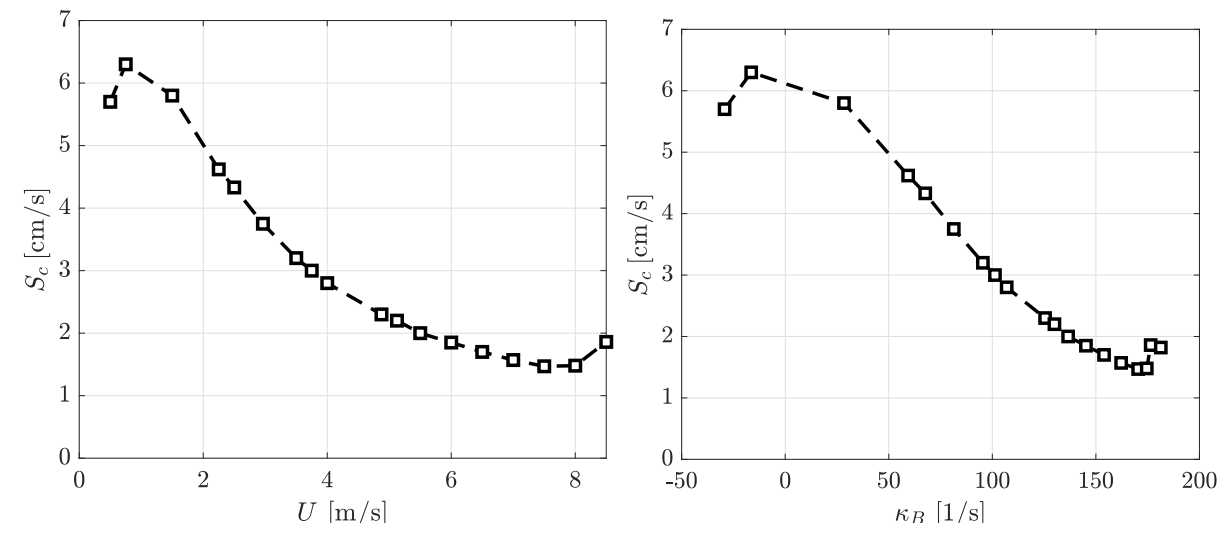

Figure 10: The consumption speed $S_{c}$ measured at the flame base versus the inflow velocity $U$ and versus the flame stretch rate at the flame base $\kappa_{B}$ for flames with $\phi=0.35$.

curvature-induced stretch is very small for this flame. For the other flames, as the inflow velocity grows, the stretch rate takes on positive values, corresponding to a diverging velocity field with extension effects on the flame surface. The maximum stretch rate for each flame is located near the flame base, and increases with $U$, reaching values as high as $180 \mathrm{~s}^{-1}$, well above the extinction strain rate associated with planar flames at $\phi=0.35$, estimated as $\kappa_{\text {ext }}=31 \mathrm{~s}^{-1}$ (see section 3 ). The stretch rate decreases gradually downstream to values below $50 \mathrm{~s}^{-1}$. A large part of the stretch rate near the flame base can be attributed to its curvature, which grows appreciably as $U$ is increased, as shown in Fig. 9b. Further downstream the curvature decays and the flames become almost parallel to the flow.

In the case of flames stabilized behind a solid body, most studies assume that the stretch experienced by the flame at the anchoring point determines the flame burning speed via the expresssion in Eq.8. In order to establish the possible correlation between the observed flame stretch rate at the flame 
base and the flame speed, we compute the flame consumption speed at the base as the integral of the heat release rate along a line normal to the flame:

$$
S_{c}=\frac{\int_{-\infty}^{\infty}\left(\rho S_{T}\right) d n}{\rho_{u}\left(T_{b}-T_{u}\right)},
$$

where $S_{T}$ is the heat release term in the energy equation (Eq. 1c) and $T_{u}$ and $T_{b}$ are the temperatures measured at the upstream and downstream end of the normal to the flame.

Figure 10 shows the flame consumption speed at the flame base as a function of the mean inflow velocity and as a function of the flame stretch rate measured at the base, showing that it decreases when the flame stretch rate grows. This would suggest, contrary to what was expected, a positive value for the Markstein length, and a flame that becomes weaker as stretch increases. The current flames are therefore able to sustain high stretch rates, much higher than the extinction strain rate that can be sustained by the equivalent planar flame. This stretch is high at the flame base, but it does not contribute to an appreciable increase of the local burning speed. On the other hand, high stretch rates near the flame base do not seem to be able to cause blow-off of these flames. As will be shown next, the current flames structure is measurably different from that of a freely propagating flame, and therefore the standard correlations between flame stretch and flame speed do not apply.

The flame presented in Fig. 11 corresponds to a mixture with $\phi=0.35$ and $U=0.5 \mathrm{~m} / \mathrm{s}$, the lowest inflow velocity for which a steady flame was obtained at this equivalence ratio. Together with the flame shape, represented by isocontours of the heat release rate corresponding to $10 \%, 30 \%$ and $50 \%$ of the maximum heat release rate, we include vectors representing the gas 

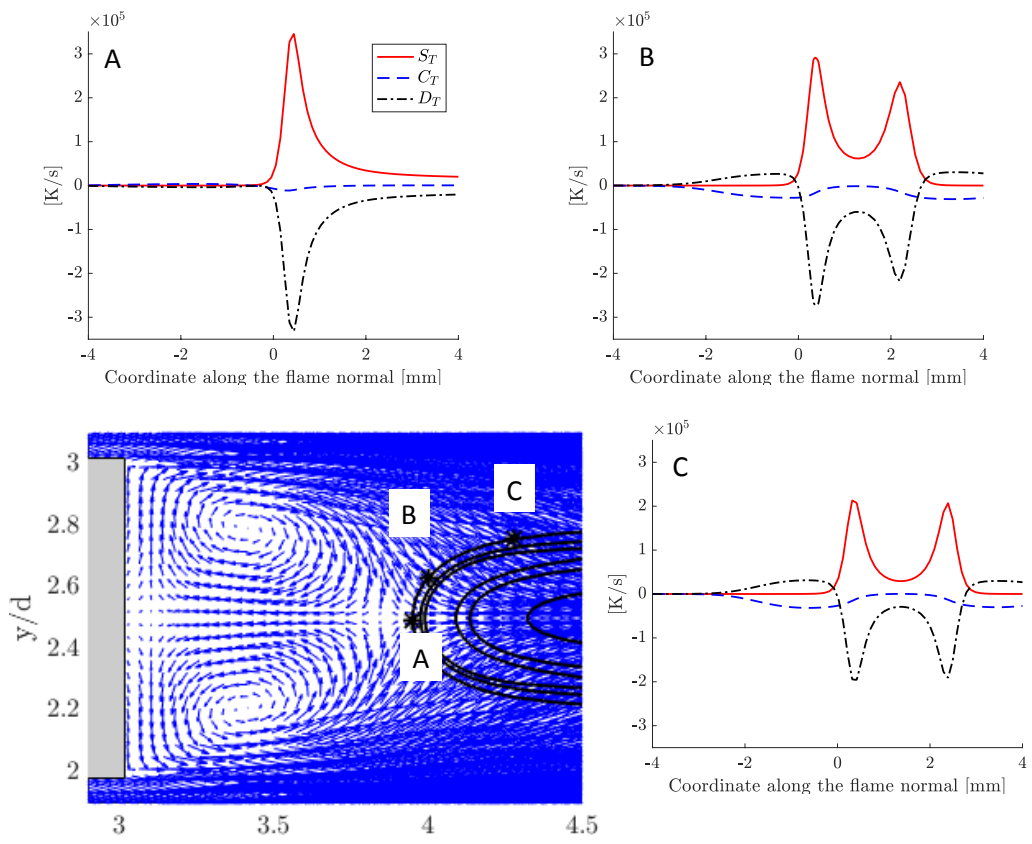

Figure 11: The flame structure as depicted by the reaction $\left(S_{T}\right)$-convection $\left(C_{T}\right)$-diffusion $\left(D_{T}\right)$ balance in the energy equation along lines normal to the flame at the flame base (A) and at two close-by locations (B and C) for a steady flame with $\phi=0.35$ and $U=0.5$ $\mathrm{m} / \mathrm{s}$. 

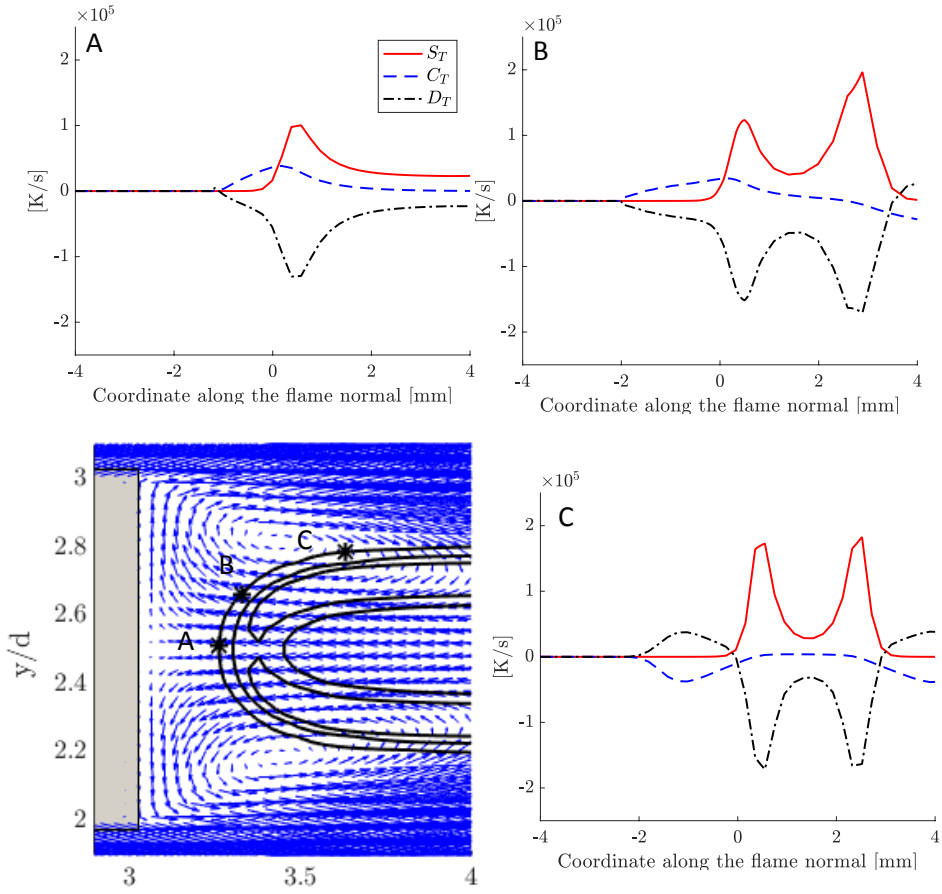

Figure 12: The flame structure as depicted by the reaction $\left(S_{T}\right)$-convection $\left(C_{T}\right)$-diffusion $\left(D_{T}\right)$ balance in the energy equation along lines normal to the flame at the flame base (A) and at two locations inside the recirculation zone (B and C) for a steady flame with $\phi=0.35$ and $U=3 \mathrm{~m} / \mathrm{s}$. 
velocity field. Plots of the balance of the reaction $\left(S_{T}\right)$, convection $\left(C_{T}\right)$ and diffusion $\left(D_{T}\right)$ terms of the energy equation (Eq.1c) along lines perpendicular to the flame at three different points in the flame surface are also included in Fig. 11. The zero value at the $x$ axis in each plot corresponds to the points marked with an asterisk along the flame surface (A, B and C). These balance plots show that the structure of the flame corresponds to the standard structure of a premixed freely propagating flame. Namely, two zones can be distinguished: 1) a preheat zone where $C_{T}<0$ and $D_{T}>0$, and therefore a temperature decrease by convection from fresh reactants is balanced by a temperature increase by diffusion from the reaction zone, is followed by 2 ) a reaction zone where reaction and diffusion are balanced. Note that while the preheat zone is barely visible in the plot corresponding to the flame base (A), since convection (and therefore diffusion) are very small there, in the two other flame structure plots, corresponding to points B and C downstream the balance of $C_{T}$ and $D_{T}$ in the $x<0$ region is clear. In the reaction zone $(x>0)$ the temperature increase related to heat release is compensated by diffusion-driven temperature decrease for the three plots.

The flame in Fig. 12 corresponds to a mixture with equivalence ratio $\phi=0.35$ and a high inflow velocity $(U=3 \mathrm{~m} / \mathrm{s})$. In this case, the flame is anchored inside the recirculation zone and, therefore, for a part of the flame surface the flow is reversed, moving from products to reactants, as clearly marked by the velocity vectors. The balance of reaction, diffusion and convection ( $S_{T}, D_{T}$ and $C_{T}$, resp.) in the energy equation is plotted along three lines normal to the flame surface, crossing it at the flame base (A), and at two locations inside the recirculation zone corresponding to flow 
going from products to reactants (B) and from reactants to products (C). The flame structure does not correspond to a standard propagating flame anymore: in the first and second balance plots (A and B), where the flow is reversed, in the preflame zone $C_{T}>0$, so that the temperature increases by convection from the reaction zone; this increase is balanced by diffusion from fresh reactants $\left(D_{T}<0\right)$. Equivalently, if we plotted terms of the species equation balance we would find that species are convected from the flame to this preflame zone, and the only way for reactants to get to the flame in this region is by diffusion. This is what is often called a negative speed flame, and it is generated by the presence of the wall, which is a stagnation surface that restrains the flame movement $[18,29]$. This flame structure was also described in [5] in their numerical study of blow-off of bluff-body stabilized flames. Further downstream along the flame surface the flow changes direction and goes from reactants to products again, and the standard propagating flame structure is recovered (see the plot corresponding to point $\mathrm{C}$ in Fig. 12).

In summary, we have seen that at the lowest inflow velocities the ultralean flame stabilizes in the low velocity region just downstream the recirculation zone. In this case the flow moves from the reactants side to the products side and the structure of the flame (depicted by plots of reactionconvection-diffusion balance in the energy equation) is the standard structure of a premixed propagating flame. As the flow velocity is raised the recirculation zone grows and the flame moves to a location closer to the flame holder, stabilizing in a low reverse velocity region. For these flames, the flow is reversed along part of the flame surface, going from the products side to the 
reactants side. The flame structure in these reversed flow parts is that of a restrained flame with negative flame speed, in which reactants reach the reaction zone by diffusion. Because of this unconventional structure the study of the flame stabilization can not rely on standard relations established for freely propagating flames, such as Eq. 8.

\subsection{Effect of preferential diffusion}

It is clear from the preceding discussion and figures that the current ultralean $\mathrm{H}_{2}-\mathrm{CH}_{4}$ flames become stronger as $U$ (and therefore the stretch rate) is increased, but also that local burning at the base is not necessarily more intense. Instead, the enhanced flame strength should be related to more intense local burning at some region close to the base, but not restricted to a point. It is well known that positive curvature of a flame together with a low Lewis number leads to enhanced burning rate because of focusing of the highly diffusive reactants in the curved region. High positive strain rate also contributes to this enhancement. We will see in this section that the focusing of $\mathrm{H}_{2}$ in the curved region near the flame tip is what makes the current flames become stronger as the inflow velocity $U$, and with it the curvature and strain, are increased.

The local equivalence ratio defined as:

$$
\phi_{l}=\frac{0.5 \sum X_{\mathrm{H}}+2 \sum X_{\mathrm{C}}}{\sum X_{\mathrm{O}}},
$$

where $X_{H, C, O}$ stand for the atomic molar fractions of $\mathrm{H}, \mathrm{C}$ and $\mathrm{O}$, is a measure of local changes in the mixture composition caused by preferential diffusion. It is used for this purpose, for example, in [7], or in the experiments of $[30,31,32]$. As fuel is convected by the flow or consumed by the chemical 

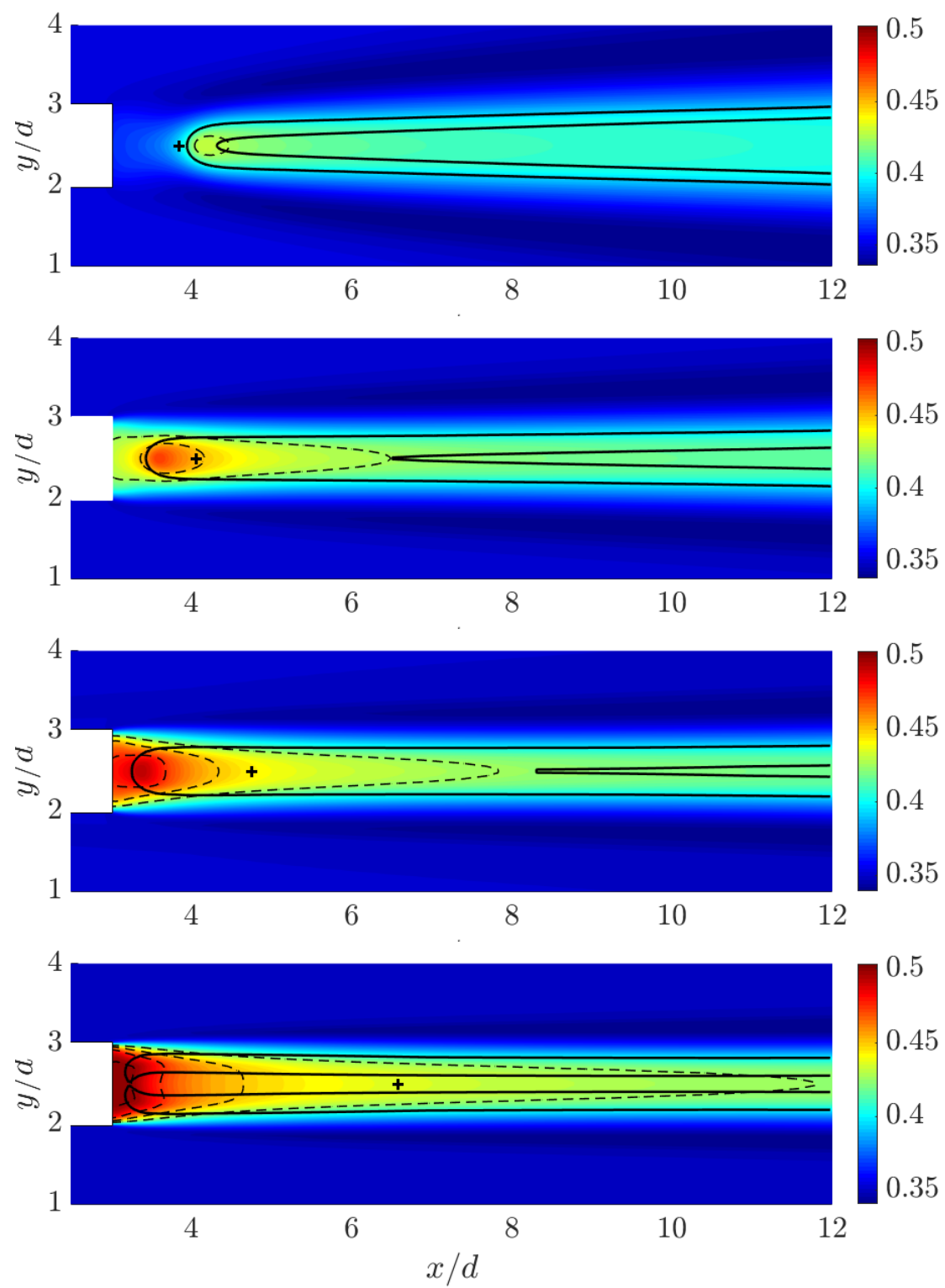

Figure 13: Contour plots representing the local equivalence ratio $\phi_{l}$ estimated via Eq. 12 for steady flames with $\phi=0.35$ and $U=0.5,1.5,3$ and $8.5 \mathrm{~m} / \mathrm{s}$. Broken lines mark the contour $\phi_{l}=0.425$. The flame surface is represented by a black solid line. 
(a)

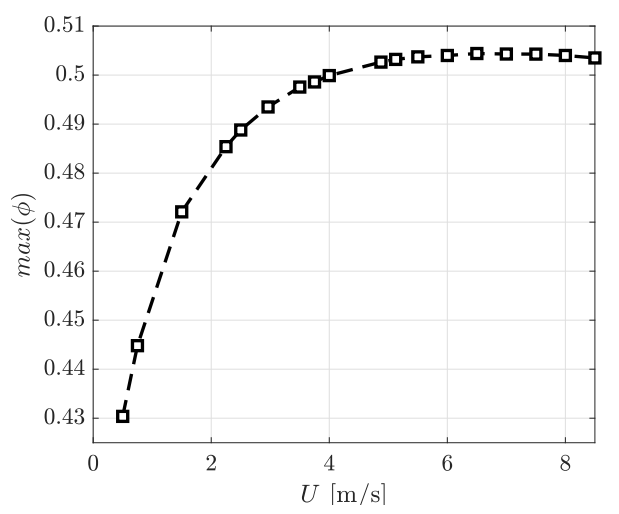

(b)

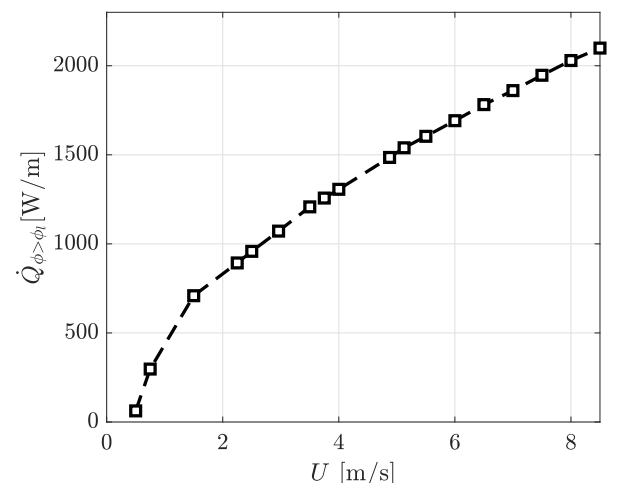

Figure 14: (a) The maximum value of the local equivalence ratio $\phi_{l}$ and (b) the heat release rate integrated in the region where $\phi_{l}$ is above the flammability limit $\left(\phi_{l} \geq 0.425\right)$ versus the inflow velocity $U$. 
reactions, $\mathrm{C}$ and $\mathrm{H}$ are conserved. A local concentration of $\mathrm{H}$ or $\mathrm{C}$ atoms different from that in the fresh reactants is therefore necessarily linked to diffusion effects. If diffusion of reactants is faster in a region, locally higher values of $\phi_{l}$ should be found. In the present simulations a local equivalence ratio above the initial value of $\phi=0.35$ in the reactants zone indicates a locally richer mixture that would burn faster. A local equivalence ratio above $\phi=0.35$ in the products region indicates products generated during the combustion of a locally richer mixture.

Figure 13 shows the local equivalence ratio, $\phi_{l}$, for four flames corresponding to the same fresh reactants initial equivalence ratio $\phi=0.35$ and different values of the mean velocity $U$, covering the full range of steady flames. For all these flames, there is a region near the curved flame base where the local mixture equivalence ratio equals to or exceeds the flammability limit $\left(\phi_{l} \geq 0.425\right)$, corresponding in the figure to the region enclosed by the broken line. This region is very small for the flame at the lowest inflow velocity $(U=0.5 \mathrm{~m} / \mathrm{s})$, for which strain-related stretch is negative but a positive curvature exists, and becomes larger as the flow velocity is increased. The maximum value of $\phi_{l}$ in the flames, plotted in Fig. 14, also increases with the flow velocity, until it reaches a saturation value at about $\phi_{l}=0.5$. The area of the region of flammable mixture increases continuously and, for the flame corresponding to the fastest flow $(U=8.5 \mathrm{~m} / \mathrm{s}$ in Fig. 13), occupies a large region of the recirculation zone. The heat release rate computed in the region where the mixture is flammable $\left(\phi_{l} \geq 0.425\right)$ is also plotted in Fig. 14, showing that it increases continuously with $U$. This locally richer mixture region, above the flammability limit, growing with the inflow velocity, ex- 
plains why the current flames become stronger and therefore closer to the flame holder as the flow velocity and the flame stretch rate are increased. It also explains why the current flames sustain a higher stretch rate than that corresponding to extinction of a planar flame with $\phi=0.35$.

To verify that in the locally richer regions described above the mole fraction of atoms of $\mathrm{H}$ predominates over the mole fraction of $\mathrm{C}$ atoms, we present in Fig. 15 scatter plots of the local ratio of $\mathrm{H}$ and $\mathrm{C}$ atoms mole fractions, $X_{\mathrm{H}} / X_{\mathrm{C}}$, scaled by its value in the fresh reactants $\left(X_{\mathrm{H}} / X_{\mathrm{C}}\right)^{0}$ against the local temperature for two steady flames with $\phi=0.35$ and $U=0.5$ and $3 \mathrm{~m} / \mathrm{s}$. This plot confirms that at intermediate temperatures, that is, in the preheating and reaction zones, the ratio of the concentrations of $\mathrm{H}$ to $\mathrm{C}$ atoms increases above its initial value in the fresh reactants (and that in the product region this ratio decreases again). This implies that, as expected, it is the fast-diffusing species, $\mathrm{H}_{2}$ (and possibly $\mathrm{H}$ ), which concentrate preferentially in the reaction region of the flame.

The anomalous behavior shown by the current ultra-lean, hydrogen containing flames is related to the high diffusivity of $\mathrm{H}_{2}$, which focuses in the highly strained and curved flame base region, and results in local combustion at higher equivalence ratios. As the inflow velocity is raised, this effect is enhanced, leading to stronger burning at the flame tip and the flame getting closer to the flame holder.

The effect of preferential diffusion of hydrogen-containing species on the local equivalence ratio was also detected experimentally $[30,31,32]$. In this series of papers, changes in the local equivalence ratio and the atom mole fraction ratios were measured in turbulent methane and hydrogen-methane 
flames stabilized behind a bluff-body. This effect was amplified when the reactants inflow velocity was raised. The authors linked this amplification to an increase in the size of the recirculation zone and the relative proximity between the flame and the recirculation zone: the effect of preferential diffusion would be accumulated or integrated over the effective residence time of the fluid in the recirculation zone. This may also explain the results in the present case: as the inflow velocity is raised, the recirculation zone grows and the flame moves closer to the flame holder; therefore the area where burning occurs inside the recirculation zone increases. The longer residence time increases the effect of high diffusivity bringing $\mathrm{H}_{2}$ towards the reaction zone, resulting in burning at locally higher equivalence ratios. The saturation in the maximum local equivalence ratio for large inflow velocity observed in Fig. 14 was also reported in [30], but no explanation could be found for it. Future exploration of flames in the present configuration could help in answering this.

\section{Conclusions}

In this work we investigated the anchoring of ultra-lean $\mathrm{H}_{2}-\mathrm{CH}_{4}$ flames behind a thin highly conducting solid. For this purpose, we used direct numerical simulations, including detailed chemistry and species transport, as well as the conjugate heat exchange with the conducting body in a planar (2D) configuration.

We reproduced experimental findings of [16], in which 'anomalous blowoff" was found for $40 \% \mathrm{H}_{2}-60 \% \mathrm{CH}_{4}$ mixtures at equivalence ratio $\phi=$ 0.35. Namely, it was found, that as the inflow reactants velocity increased, 
(a)

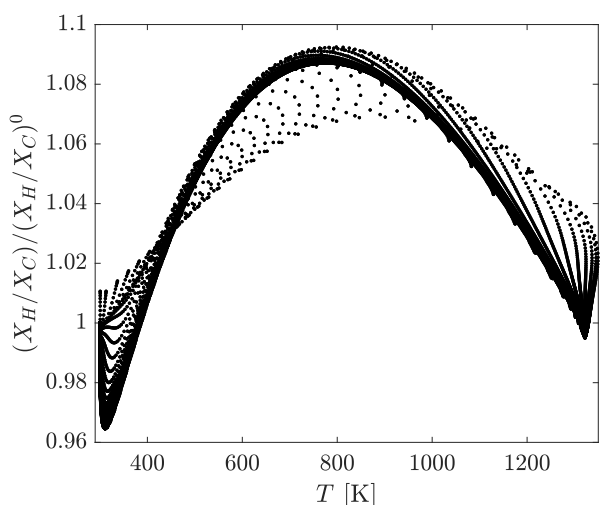

(b)

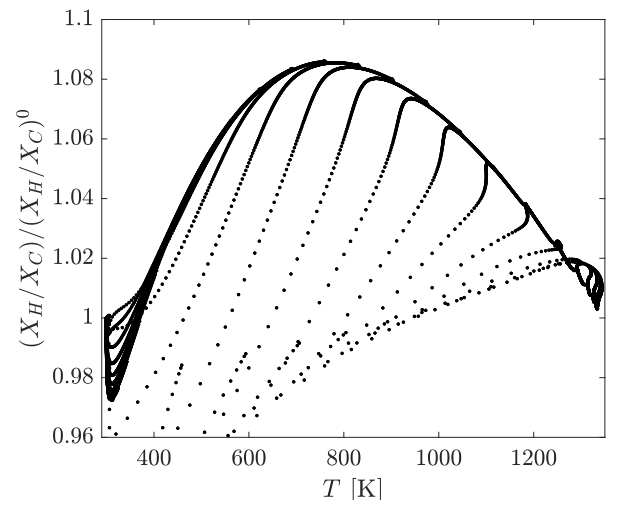

Figure 15: Scatter plots showing the correlation between the ratio of $\mathrm{H}$ to $\mathrm{C}$ atoms, scaled with the ratio measured in the fresh reactants, $\frac{X_{\mathrm{H}} / X_{\mathrm{C}}}{\left(X_{\mathrm{H}} / X_{\mathrm{C}}\right)^{0}}$ and the local temperature $T$ for flames with $\phi=0.35$ and $U=0.5 \mathrm{~m} / \mathrm{s}$ (a) and $3 \mathrm{~m} / \mathrm{s}$ (b). 
the flame stabilized closer to the flame holder. Conversely, as the inflow velocity was reduced, the steady flame moved farther away from the flame holder, and for a sufficiently small value of this velocity the flame was blownoff. This phenomenon, anomalous in that it is opposite to that previously described for pure methane flames, was shown to be related to the high diffusivity of $\mathrm{H}_{2}$, which result in $\mathrm{H}_{2}$ concentrating on the highly strained and curved flame base, and leading to local burning at richer equivalence ratios. As the inflow rate is increased, flame stretch near the flame base is increased and this focusing effect is enhanced, leading to stronger burning at the flame base and it moving closer to the flame holder, anchoring inside the flow recirculation zone. This is in contrast to the methane flames in [4], in a similar configuration, which were shown to always stabilize outside the recirculation zone. When the inflow rate is reduced below a certain limit there is not enough stretch at the flame base to generate a locally richer mixture region. Because of this combination of transport and redistribution, the current flames could not be blown-off by increasing the inflow reactants velocity, which always resulted in stronger flames.

We note that, while the present results show that thermal losses to the solid are not significant in the blow-off of the present stationary flames, this was not known a priori. For this reason conjugate heat exchange was included in the formulation of the problem. Moreover, ongoing work shows that for certain, very lean flames stabilized behind the same conducting, long solid, heat exchange with the solid could be fundamental in the stabilization and blow-off mechanisms.

We should mention that our results correspond to a highly conducting, 
short, cooled flame holder (quasi-isothermal), which does not model quantitaively the experimental longer flame holder [16]. Nevertheless, we still observe the phenomenon of "anomalous" flame blow-off.

\section{Acknowledgements}

This work was supported partly by a MIT-Technion fellowship to Dan Michaels, partly by KAUST grant number KUS-110-010- 01, and partly by the MINECO/FEDER grant number ENE2015-65852-C2-2-R.

\section{References}

[1] C. Sung, C. Law, A. Umemura, On adiabatic stabilization of inverted flames, Symposium (International) on Combustion 24 (1992) 205 - 212.

[2] T. Kawamura, K. Asato, T. Mazaki, T. Hamaguchi, H. Kayahara, Explanation of the blowoff of inverted flames by the area-increase concept, Combustion and Flame 35 (1979) 109 - 116.

[3] T. Kawamura, K. Asato, T. Mazaki, Reexamination of the blowoff mechanism of premixed flames-inverted flames, Combustion and Flame $45(1982) 225-233$.

[4] K. S. Kedia, A. F. Ghoniem, Mechanisms of stabilization and blowoff of a premixed flame downstream of a heat-conducting perforated plate, Combustion and Flame 159 (2012) 1055 - 1069.

[5] K. S. Kedia, A. F. Ghoniem, The anchoring mechanism of a bluff-body stabilized laminar premixed flame, Combustion and Flame 161 (2014) $2327-2339$. 
[6] K. S. Kedia, A. F. Ghoniem, The blow-off mechanism of a bluff-body stabilized laminar premixed flame, Combustion and Flame 162 (2015) $1304-1315$.

[7] D. Michaels, A. F. Ghoniem, Impact of the bluff-body material on the flame leading edge structure and flame-flow interaction of premixed CH4/air flames, Combustion and Flame 172 (2016) $62-78$.

[8] B. Lewis, G. von Elbe, Stability and structure of burner Flames, J. Chem. Phys. 11 (1943) 75-97.

[9] B. Lewis, G. von Elbe, Combustion, Flames and Explosions of Gases, 2 ed., Academic Press, New York, 1961.

[10] C. Treviño, S. Donnerhack, N. Peters, LDA measurements in the premixed $\mathrm{v}$ flame stabilized in the wake of a flat plate boundary layer, Combustion and Flame 85 (1991) $505-510$.

[11] S. J. Shanbhogue, S. Husain, T. Lieuwen, Lean blowoff of bluff body stabilized flames: Scaling and dynamics, Progress in Energy and Combustion Science 35 (2009) 98 - 120.

[12] F. Duchaine, A. Corpron, L. Pons, V. Moureau, F. Nicoud, T. Poinsot, Development and assessment of a coupled strategy for conjugate heat transfer with large eddy simulation: Application to a cooled turbine blade, International Journal of Heat and Fluid Flow 30 (2009) 1129 1141.

[13] C. Koren, R. Vicquelin, O. Gicquel, Self-adaptive coupling frequency 
for unsteady coupled conjugate heat transfer simulations, International Journal of Thermal Sciences 118 (2017) 340 - 354 .

[14] D. Michaels, S. Shanbhogue, A. Ghoniem, The impact of reactants composition and temperature on the flow structure in a wake stabilized laminar lean premixed CH4/H2/air flames; mechanism and scaling, Combustion and Flame 176 (2017) 151 - 161.

[15] M. Miguel-Brebion, D. Mejia, P. Xavier, F. Duchaine, B. Bedat, L. Selle, T. Poinsot, Joint experimental and numerical study of the influence of flame holder temperature on the stabilization of a laminar methane flame on a cylinder, Combustion and Flame 172 (2016) 153 - 161.

[16] Y. Shoshin, R. J. M. Bastiaans, L. P. H. de Goey, Anomalous blow-off behavior of laminar inverted flames of ultra-lean hydrogen-methane-air mixtures, Combustion and Flame 160 (2013) 565 - 576.

[17] V. Kurdyumov, Y. Shoshin, L. de Goey, Structure and stability of premixed flames stabilized behind the trailing edge of a cylindrical rod at low lewis numbers, Proceedings of the Combustion Institute 35 (2015) $981-988$.

[18] C. K. Law, Dynamics of stretched flames, Symposium (International) on Combustion 22 (1988) $1381-1402$.

[19] T. Poinsot, D. Veynante, Theoretical and Numerical Combustion, 2 ed., R.T. Edwards, 2005.

[20] K. S. Kedia, C. Safta, J. Ray, H. N. Najm, A. F. Ghoniem, A secondorder coupled immersed boundary-SAMR construction for chemically 
reacting flow over a heat-conducting cartesian grid-conforming solid, Journal of Computational Physics 272 (2014) 408 - 428.

[21] B. M. Bride, S. Gordon, M. Reno, Coefficients for calculating thermodynamic and transport properties of individual species, Technical Report NASA TM-4513, NASA, 1993.

[22] P. Paul, DRFM: a new package for the evaluation of gas-phase transport properties, Technical Report SAND-98-8203, Sandia National Labs, Livermore, CA, USA, 1997.

[23] C. Safta, J. Ray, H. N. Najm, A high-order low-Mach number AMR construction for chemically reacting flows, Journal of Computational Physics 229 (2010) $9299-9322$.

[24] M. Smooke, I. Puri, K. Seshadri, A comparison between numerical calculations and experimental measurements of the structure of a counterflow diffusion flame burning diluted methane in diluted air, Symposium (International) on Combustion 21 (1988) 1783 - 1792.

[25] R. J. Kee, F. M. Rupley, J. A. Miller, CHEMKIN-II: A FORTRAN chemical kinetics package for the analysis of gas-phase chemical kinetics, Technical Report SAND-89-8009, Sandia National Labs, Livermore, CA, USA, 1989.

[26] M. Matalon, B. J. Matkowsky, Flames as gasdynamic discontinuities, Journal of Fluid Mechanics 124 (1982) 239259.

[27] P. Clavin, G. Joulin, Premixed flames in large scale and high intensity 
turbulent flow., Journal de physique. Lettres 44 (1983) 1-12. Cited By 79.

[28] S. M. Candel, T. J. Poinsot, Flame stretch and the balance equation for the flame area, Combustion Science and Technology 70 (1990) 1-15.

[29] P. A. Libby, F. A. Williams, Structure of laminar flamelets in premixed turbulent flames, Combustion and Flame 44 (1982) 287 - 303.

[30] R. S. Barlow, M. J. Dunn, M. S. Sweeney, S. Hochgreb, Effects of preferential transport turbulent bluff-body-stabilized lean premixed $\mathrm{CH} 4 / \mathrm{H} 2$ flames, Combustion and Flame 159 (2012) 2563 - 2575.

[31] M. J. Dunn, R. S. Barlow, Effects of preferential transport and strain in bluff body stabilized lean and rich premixed CH4/air flames, Proceedings of the Combustion Institute 34 (2013) 1411 - 1419.

[32] R. S. Barlow, M. J. Dunn, G. Magnotti, Preferential transport effects in premixed bluff-body stabilized CH4/H2 flames, Combustion and Flame $162(2015) 727-735$. 